\title{
Phase resolved spectral analysis of 25 millisecond gamma-ray pulsars with the Fermi-LAT
}

\author{
Nicolas Renault-Tinacci* \\ AIM, Service d'Astrophysique, CEA Saclay, 91191 Gif Sur Yvette, France \\ Institut d'Astrophysique de Paris, UMR7095 - CNRS, Université Pierre E Marie Curie, 98 bis \\ boulevard Arago F-75014 Paris, France \\ E-mail: n.renault.tinacci@gmail.com \\ Isabelle Grenier \\ AIM, Service d'Astrophysique, CEA Saclay, 91191 Gif Sur Yvette, France \\ E-mail: isabelle.grenier@cea.fr
}

\section{Alice K. Harding}

Astrophysics Science Division, NASA Goddard Space Flight Center, Greenbelt, MD, USA

E-mail: alice.k.harding@nasa.gov

\begin{abstract}
Millisecond pulsars (MSPs) are a growing class of gamma-ray emitters. Spectral analyses of their pulsed emission bring important constraints to the theoretical models which describe the electromagnetic processes responsible for high-energy radiations in pulsar magnetosphere. The gamma-ray data collected during five years of Fermi Large Area Telescope (LAT) observations have allowed in-depth phase resolved spectral analyses of 25 of the brightest objects in the 50 $\mathrm{MeV}$ to $170 \mathrm{GeV}$ energy band. The sample exhibits a significant evolutionary sequence in spectral energy distribution of the total pulsed emission. The sequence relates three spectral parameters, namely the spectral index at low energy, the apex energy at which the maximum energy flux is emitted, and the cut-off energy, with the spin-down power of the neutron star. As the latter increases, the spectral energy distribution broadens, softens, and shifts in energy. This evolution is primarily driven by the emission in the caustic peaks. Spectral parameters change with phase present systematic patterns. Pulsars with aligned gamma-ray and radio peaks tend to be particularly luminous and soft. The sequence highlights an important transition in MSP evolution near a spin-down power of $10^{27} \mathrm{~W}$ and the possible onset of a soft emission component in addition to curvature radiation. The phase dependence of the radiative efficiency also suggests that multiple emitting regions, with different levels of electric field screening, contribute to the total pulsed gamma-ray emission.
\end{abstract}

The 34th International Cosmic Ray Conference,

30 July- 6 August, 2015

The Hague, The Netherlands

\footnotetext{
* Speaker.
} 


\section{Introduction}

The launch of the Fermi Large Area Telescope in 2008 has opened a new window onto the physics of pulsars and revolutionized the field by discovering to date $>160$ pulsars including a rapidly growing fraction of millisecond pulsars (MSPs) emitting at GeV energies. MSPs are analogous to normal pulsars (NPs) in the sense that they emit pulsed radiation from the radio to $\gamma$-ray band but are fainter. Both possess roughly equal magnetic field strength at light cylinder due to the greater compactness of MSPs magnetosphere and despite their lower magnetic field strength at surface. Recent studies (in particular 1) have shown important similarities between MSP and NP $\gamma$-ray profiles. Those two arguments point toward the existence of comparable electro-dynamic behaviours in MSP magnetosphere as in NPs.

Phase-resolved spectroscopy allows to explore the evolution of the particle distributions between different zones of the magnetosphere and/or in different parts of the accelerating gaps as their radiation sweeps past us (2). It can be used to follow spectral variations across the wings of the main peaks in order to constrain emission characteristics at different altitudes along the bundle of last open field lines where accelerating gaps are thought to exist.

We have taken advantage of five years of Fermi-LAT $\gamma$-ray data to analyze the spectra of 25 of the brightest millisecond $\gamma$-ray pulsars. We report on the results obtained for their total emission, across the rotational phase and the population trends in the sample.

\section{Millisecond pulsars sample}

To ensure large enough photon statistics to allow phase-resolved spectroscopic analyses, the sample includes objects bright and also bright enough with respect to the interstellar background in the source direction. With this criterion, the sample tends to span at best the same range than MSP population in period $P$ and $\dot{P}$, spin-down power, magnetic field strength at light cylinder, obliquities (3) and galactic coordinates. The sample also covers a large variety of $\gamma$-ray light-curve morphologies with broad or sharp peaks, with pulsed emission over the whole phase or not, with bridge emission or not, and with different numbers of peaks. We categorize them as ramps (RA) and multi-peak pulsars. The latter is divided into 3 peaks (3P), 2 peaks (2P), dome+peak (DP). A dome+peak light curve consists of a broad pulse followed by a narrow one. A ramp corresponds to a single broad pulse with either a gentle rise and a steep decrease, or a steep rise and a gentle decrease.

\section{Fermi-LAT observations and analyses}

We have used 5 years of Pass 7 reprocessed Fermi-LAT data and taken into account the LAT instrument response functions ${ }^{1}$ with the associated diffuse background templates (P7REP_V15CLEAN). We have selected photon within the energy range $50 \mathrm{MeV}-172 \mathrm{GeV}$ for spectral analysis excluding higher energies because of poorer statistics. We have kept photons within a radius of 18 degrees from the pulsar position and excluded the periods when the pulsar was viewed at zenith angles $>100^{\circ}$ to minimize contamination by Earth limb photons.

\footnotetext{
${ }_{1}$ available at the Fermi Science Support Centre: http://fermi .gsfc.nasa.gov/ssc/
} 
We have made use of the exceptional stability of MSPs rotation to gather more data by extending the timing solution of each pulsar to the actual data time interval, beyond the timing validity period. The ephemerides are provided by different teams within the Fermi-LAT Collaboration.

In this analysis, we have systematically selected the phase intervals for the phase-resolved spectroscopy in a way that would preserve the morphological structures of $\gamma$-ray profiles while keeping a minimum number of photons per interval.

The spectral analysis was performed for each phase interval as well as for the total emission. The spectral fit with the LAT data was performed by means of a binned maximum-likelihood estimator with Poisson statistics. We stress that the present analysis independently fits the pulsar flux in each energy band and that it does not require any prior assumption on the spectral shape.

We have characterized the resulting photon spectra by a single power law with a photon index $\Gamma$, or by a power law with an exponential cut-off with a cut-off energy $E_{\text {cut }}$. The cut-off energy gives a measure of either the maximum particle energy in the radiation reaction limit, or the maximum particle energy relative to the maximum $E_{\|}$if the photons do not pair produce. The photon index provides indirect information on the primary particle spectra and pile-up in phase of emissions produced in different locations (in particular in the caustic regions). We derived $\Gamma$ and $E_{\text {cut }}$ from a bivariate fit to the photon spectra. The fit uses a maximum-likelihood estimator with a Gaussian probability density function in flux, and a uniform probability in energy to account for the broad width of the energy bands. We have also estimated the apex energy, $E_{\text {apex }}$, at which the spectral energy distribution (SED) reaches a maximum flux by fitting a second-order polynomial with a $\chi^{2}$ minimization around the maximum of each SED.

\section{Results}

We have looked for population trends for total emission and across phase in the hardness and cut-off energy of the emission, in the characteristic apex energy, and in luminosity. A number of patterns emerge which we summarize below.

\section{MSP spectral sequence:}

The phase-averaged SEDs all peak near $1 \mathrm{GeV}$, but they significantly differ from one another. They span over more than a decade in apex energy, from 0.3 to $5 \mathrm{GeV}$. They also exhibit a large variety of slopes below the apex energy. Some SEDs steeply rise to the apex while others are rather flat down the lowest energies observed by the LAT.

Figure 1 shows how the apex energy, $E_{\text {apex }}$, and the low-energy photon index, $\Gamma$, vary respectively with $\dot{E}$ and the magnetic field strength, $B_{\mathrm{LC}}$, at the light cylinder. We detect a significant increase in photon index $B_{\mathrm{LC}}$ (and $\dot{E}$ ) for all pulsars and a significant (with non-parametric pairwise-slope statistics from 4) concave relation of the apex energy with $\dot{E}$ (and $B_{\mathrm{LC}}$ ). A constant or linear variation is rejected with a confidence probability of hardly $100 \%$. Parabolic fits to the data points indicate that the apex energy culminates at about $2.5 \mathrm{GeV}$ near a spin-down power of $10^{27} \mathrm{~W}$. The MSP magnetospheres therefore deliver their maximum radiative flux at an energy which varies with the rotational power of the neutron star.

While confronting the spectral characteristics of the total emission with the radio-to- $\gamma$-ray lag (see Fig 1: in bottom panels and colours in top panels), a marked difference has emerged: pulsars with aligned radio and $\gamma$-ray pulses emit significantly softer $\gamma$-rays (i.e., larger $\Gamma$ and lower $E_{\text {apex }}$ ) 

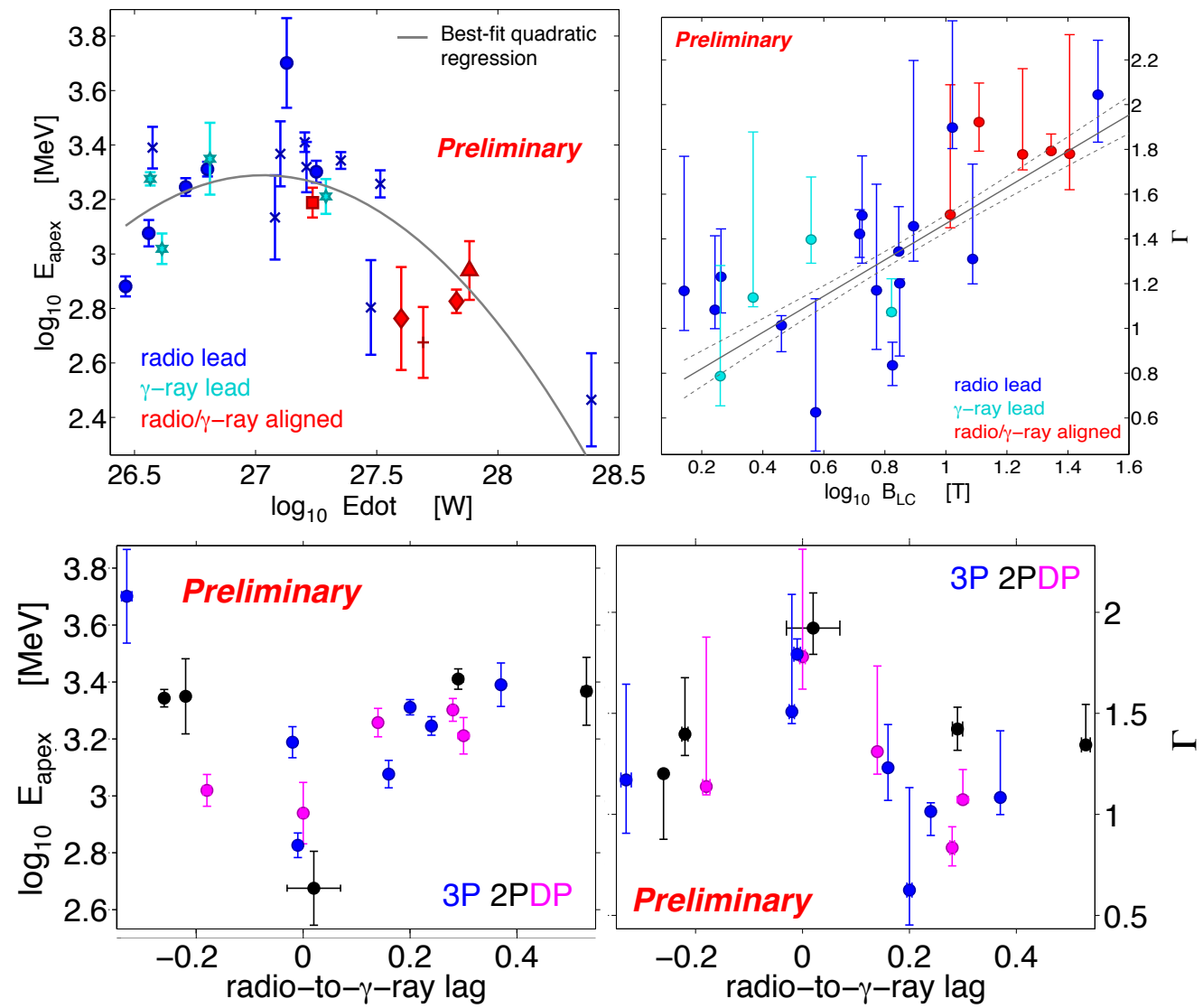

Figure 1: Top panels show the distributions of $E_{\text {apex }}$, and $\Gamma$ of the total emission respectively as function of spin-down power, $\dot{E}$, and of magnetic field strength at the light cylinder, $B_{\mathrm{LC}}$. The solid and dashed lines give the best-fit regression and standard deviation. Colours and markers code the lag between radio and $\gamma$-ray profiles (blue circle when the radio leads $\gamma$-ray, cyan square when $\gamma$-ray leads and red cross when radio and $\gamma$-ray are aligned). Bottom panels exhibits the distributions of the $E_{\text {apex }}$ and $\Gamma$ of the total pulsed emission as a function of radio-to- $\gamma$-ray lag. Colours denote the morphological types of the multi-peaked light curves.

than the others however we cross the caustic. (3) have noticed that these objects have unusually large spin-down powers, hence high magnetic field strengths near the light cylinder.

For each pulsar in the data set, we have calculated the curvature radiation $(\mathrm{CR})$ produced by primary electrons located near the light cylinder, with a radius of curvature for their trajectory equal to the light-cylinder radius (5), and with different power-law distributions in Lorentz factors. We have looked for trends with $\dot{E}$ in the inferred CR spectra across the Fermi-LAT energy band. For a very large variety of input electron spectra, CR leads to trends at odds with the observations. The observed variations (increase in $\Gamma$ and rise and fall of $E_{\text {apex }}$ with $\dot{E}$ ) therefore suggest the onset or amplification from the least to most energetic MSPs of sub-GeV emission from a subset of primary particles to broaden and soften the total SED. Few hypotheses can explain this softer component, in particular the softening coincident with the radio/ $\gamma$-emission alignment points toward a synchrotron radiation from heated primaries which can gain a pitch angle by cyclotron resonant absorption of radio photons (6). A smooth transition from $E_{\|} \neq 0$ to $E_{\|}=0$ due to a varying screening in the transfield direction in outer gap (7) can produce $\mathrm{CR}$ at few hundreds $\mathrm{MeV}$ providing the 
required softer emission. Inspection of the spectra across phase suggest that the global softening of the whole emission is driven by the peaks aligned in phase with the radio, but this point needs confirmation with more data.

On the other hand, (8) estimated by equating the pair-starved and screened potentials that the critical $\dot{E}$ between the two regimes is about $10^{27} \mathrm{~W}$ which is in line with the critical $\dot{E}$ observed in the top left panel of Fig. 1. Thus it would suggest that the rise and fall would indicate this regime change, where screening starts to dominate above roughly $10^{27} \mathrm{~W}$.

\section{Radiative efficiency evolution across phase:}

We have computed the $\gamma$-ray luminosities above $50 \mathrm{MeV}$ of the total emission and in individual phase intervals and fitted power laws between the $\gamma$-ray luminosities and spin-down powers. The whole set of luminosities for total emission approximately scales with $\dot{E}$ with a large scatter. It is consistent with the larger MSP sample in (9). However we observe that ramps rather tightly follow a steep $L_{\gamma} \propto \dot{E}^{1.34 \pm 0.13}$ relation. The rest of the pulsars, which exhibit multi-peaked profiles, follows an $L_{\gamma} \propto \dot{E}^{0.59 \pm 0.15}$ relation close to the $\sqrt{\dot{E}}$ dependence known to characterize young pulsars which efficiently screen their parallel electric fields in thin gaps. .

Figure 2 shows the evolution with $\dot{E}$ of the luminosities recorded in the same relative phase intervals in the case of multiple peaks. The present data interestingly suggest different evolutions in the different parts of the light curves. The luminosities produced in the outer wings, namely the leading wing of the first peak (P1L) and the trailing wing of the second peak (P2T), appear to scale with $\dot{E}$. Conversely, the luminosity evolution in the cores of the peaks and in their inner wings,

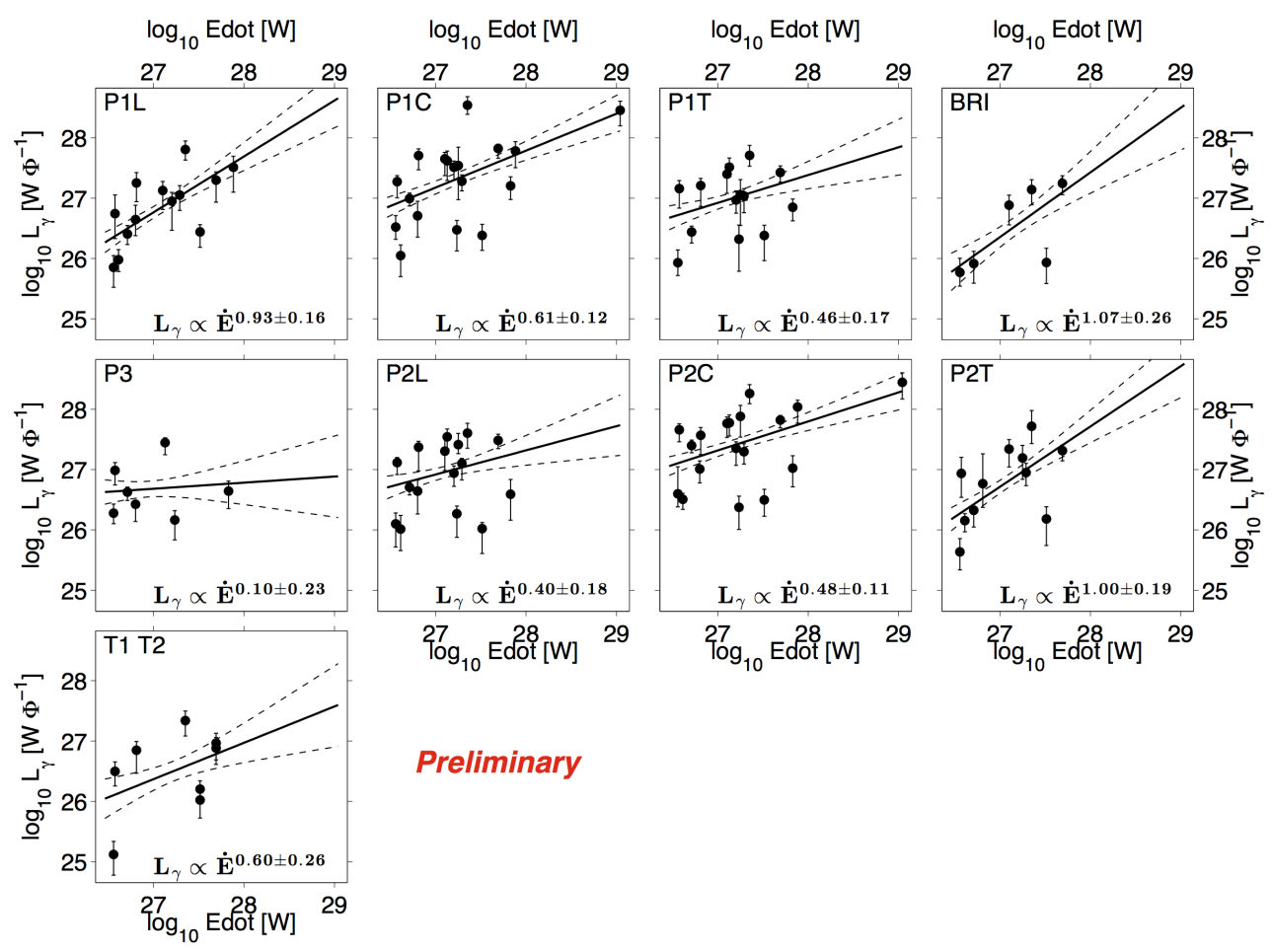

Figure 2: Evolution of the $\gamma$-ray luminosity as a function of spin-down power in relative phase intervals for multiple-peak pulsars. Solid and dashed lines respectively give the best-fit power law and standard deviation. 
namely the trailing wing of the first peak (P1T) and the leading wing of the second peak (P2L), is consistent with a $\sqrt{\dot{E}}$ dependence. So is the evolution of the faint tail emission arising well outside the main peaks. The data suggest a steeper $(\propto \dot{E})$ trend in the bridge than in the adjacent inner wings. The luminosity of the third peak shows no significant variation. These trends suggest that the emission seen outside the main peaks arises from particles accelerated in regions of unscreened parallel electric field, while the emission produced in and between the bright peaks originate from particles accelerated in thin screened gaps.

The same study for the pulsed ramps shows much less scatter in the distribution than for multiple-peak pulsars but the trends in all phase intervals are consistent with the scaling of the total luminosity as $\dot{E}^{1.34 \pm 0.13}$. It suggests that the accelerating electric fields, $E_{\|}$, are not efficiently screened for ramp pulsars, i.e. the pair multiplicity is small, and thus that the maximum energy of the pairs does not saturate near $\mathrm{TeV}$ energies in these objects.

\section{Spectral changes with phase:}

Figures 3 compare the spectral parameters measured in the cores of the two main peaks. To trace relative differences, we have plotted the ratios of apex energies and the difference in photon index as a function of the $>50 \mathrm{MeV}$ energy flux ratio between the two emissions. We have also added the information about the total $\gamma$-ray luminosity emitted $>50 \mathrm{MeV}$ in both cores to trace the total activity of the pulsar. Figures 3 suggest mild correlations between the relative energy flux and relative apex energy or photon index, with respective correlation coefficients of 0.71 and -0.74 . These connections together with the distribution of the ratio/difference with indicate that the brighter the peak core, the higher its apex energy, the harder the SED at low $\gamma$-ray energies (lower $\Gamma$ i.e. steeper rise of the SEDs up to $E_{\text {apex }}$ ), irrespective of their order in the light curve.

The correlation between the ratio of energy flux and $E_{\text {apex }}$ can be simply explained by the dominance of $\mathrm{CR}$ for which a higher flux is accompanied by a larger $E_{\text {apex }}$. Additionally, due to caustic effects $(10 ; 11 ; 2 ; 12)$, summation of emission components originating from a wide or limited range in altitude (thus with different $E_{\|}$or $\rho_{\mathrm{LC}}$ ) may produce respectively broad or narrow SED characterized by a larger or smaller $E_{\text {apex }}$ and thus explain the relative photon index correlation.

The trajectories of the particles moving outward in the magnetosphere are straighter along trailing field lines than along leading ones, at any altitude up to the light cylinder in the observer inertial frame $(5 ; 13)$. Because of this geometry and because curvature radiation (CR) is the primary radiation process to produce the observed high-energy $\gamma$ rays, it has been argued that particle acceleration at high altitude in the outer gap, slot gap, or pair-starved open magnetospheres should result in harder CR emission in the trailing peak (5). This idea has been systematically verified for un-recycled young pulsars (e.g., 2; 14) at odds with the studied MSPs. Quantitative frequencies of the position of the hardest peak, estimated in the case of a magnetosphere with force-free conditions inside the light cylinder and a finite conductivity outside (FIDO, 13) compare well with the values obtained for the current MSP sample. The hardness difference between the two $\gamma$-ray peaks and the present finding of brighter and harder first peaks may hold a key to solve the long debate between single-pole and two-poles models.

Finally the evolution of spectral parameters across phase has revealed some systematic patterns. Spectra are generally softer (lower $E_{\text {apex }}$ and larger $\Gamma$ ) outside the main peak cores exhibiting a potential link between hardness and caustic effects. Ramp spectral studies hint that for some 

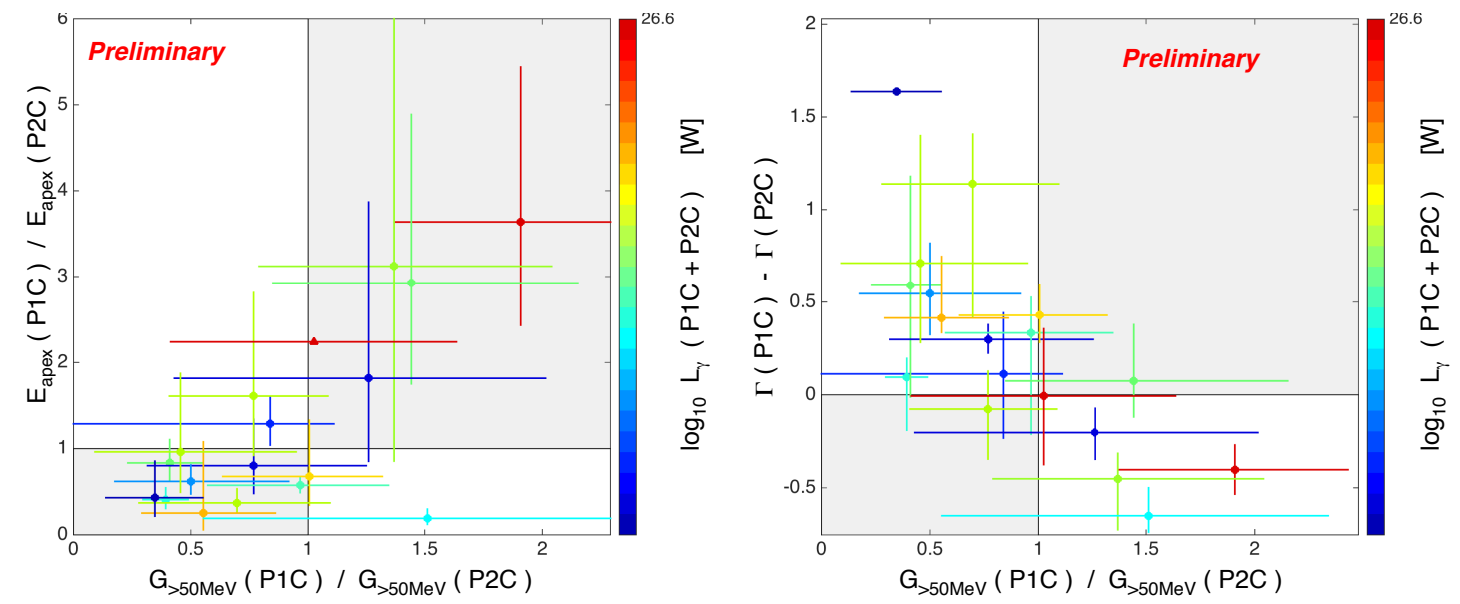

Figure 3: Left panel: ratios of the apex energies in the cores of the two peaks as a function of their energetic fluxes above $50 \mathrm{MeV}$ ratios. The grey areas indicate the regions where both ratios are either $>1$ or $<1$. Right panel: differences of the photon indices in the cores of the two peaks as a function of their energetic fluxes above $50 \mathrm{MeV}$. The grey areas indicate the regions where the difference $>0$ or where the difference $<0$. For both panels, colours denote the total $\gamma$-ray luminosities in the cores.

ramps, possibly the most asymmetric, apex energy increases with the ramp photon count while the possible most symmetric present barely no spectral variation. We have noted that the bridge emission in the compact magnetospheres of MSPs appears to be much softer than in the young pulsars.

\section{Conclusion}

All results would benefit more statistics for confirmation and some observations, particularly the spectral patterns with phase, still remain puzzling. But they point to a picture, beyond that of only thin caustic gaps or opened accelerating regions above polar caps, where a pulsar magnetosphere can sustain accelerating regions with different screening properties in distinct regions. In this context, emission from both types of regions may combine or not in the light curve depending on the observer angle. Possible locations for these different regions are near the current sheet and last open field lines, often referred to for thin screened gaps, and above the polar caps. The spectral sequence we detect in the sample (change in apex energy and slope with $\dot{E}$ ) highlights an important transition in MSP evolution near $\dot{E} \sim 10^{27} \mathrm{~W}$ with the onset or enhancement of a soft emission component from the primary particles, in addition to curvature radiation which seems to be still dominant. The "brighter, the harder the peak core" result provides a new diagnostic to enlighten the pulsar model comparisons.

\section{Acknowledgments}

The Fermi-LAT Collaboration acknowledges generous ongoing support from a number of agencies and institutes that have supported both the development and the operation of the LAT as well as scientific data analysis. These include the National Aeronautics and Space Administration and the Department of Energy 
in the United States, the Commissariat à l'Energie Atomique and the Centre National de la Recherche Scientifique / Institut National de Physique Nucléaire et de Physique des Particules in France, the Agenzia Spaziale Italiana and the Istituto Nazionale di Fisica Nucleare in Italy, the Ministry of Education, Culture, Sports, Science and Technology (MEXT), High Energy Accelerator Research Organization (KEK) and Japan Aerospace Exploration Agency (JAXA) in Japan, and the K. A. Wallenberg Foundation, the Swedish Research Council and the Swedish National Space Board in Sweden. Additional support for science analysis during the operations phase is gratefully acknowledged from the Istituto Nazionale di Astrofisica in Italy and the Centre National d'Études Spatiales in France. The authors gratefully acknowledge the Pulsar Search and Timing Consortia.

\section{References}

[1] A. A. Abdo, M. Ajello, A. Allafort, et al., The Second Fermi Large Area Telescope Catalog of Gamma-Ray Pulsars, ApJS 208 (Oct., 2013) 17, [arXiv: 1305. 4385]. (2 ${ }^{\text {nd }}$ Fermi Pulsar Catalog, 2PC).

[2] A. A. Abdo, M. Ackermann, M. Ajello, et al., The Vela Pulsar: Results from the First Year of Fermi LAT Observations, ApJ 713 (Apr., 2010) 154-165, [arXiv: 1002 . 4050]. (Vela Pulsar 11 months).

[3] T. J. Johnson, C. Venter, A. K. Harding, et al., Constraints on the Emission Geometries and Spin Evolution of Gamma-Ray Millisecond Pulsars, ApJS 213 (July, 2014) 6, [arXiv: 1404 . 2264].

[4] J. Abrevaya and W. Jiang, Pairwise-slopes statistics for testing curvature, 2003.

[5] K. Hirotani, Pulsar Outer-gap Electrodynamics: Hardening of Spectral Shape in the Trailing Peak in the Gamma-Ray Light Curve, ApJ 733 (June, 2011) L49, [arXiv: 1104 . 5357].

[6] A. K. Harding, J. V. Stern, J. Dyks, and M. Frackowiak, High-Altitude Emission from Pulsar Slot Gaps: The Crab Pulsar, ApJ 680 (June, 2008) 1378-1393, [arXiv: 0803 . 0699].

[7] Y. Wang, J. Takata, and K. S. Cheng, Gamma-ray Spectral Properties of Mature Pulsars: A Two-layer Model, ApJ 720 (Sept., 2010) 178-190, [arXiv: 1007 . 0609].

[8] A. K. Harding, A. G. Muslimov, and B. Zhang, Regimes of Pulsar Pair Formation and Particle Energetics, ApJ 576 (Sept., 2002) 366-375, [astro-ph/0205077].

[9] A. A. Abdo, M. Ajello, A. Allafort, et al., The Second Fermi Large Area Telescope Catalog of Gamma-Ray Pulsars, ApJS 208 (Oct., 2013) 17, [arXiv: 1305. 4385].

[10] M. Morini, Inverse Compton gamma-ray from pulsars. I - The VELA pulsar, MNRAS 202 (Feb., 1983) 495-510.

[11] J. Dyks and B. Rudak, Two-Pole Caustic Model for High-Energy Light Curves of Pulsars, ApJ 598 (Dec., 2003) 1201-1206, [astro-ph/].

[12] A. K. Harding, J. V. Stern, J. Dyks, and M. Frackowiak, High-Altitude Emission from Pulsar Slot Gaps: The Crab Pulsar, ApJ 680 (June, 2008) 1378-1393, [arXiv: 0803. 0699].

[13] C. Kalapotharakos, A. K. Harding, and D. Kazanas, Gamma-Ray Emission in Dissipative Pulsar Magnetospheres: From Theory to Fermi Observations, ApJ 793 (Oct., 2014) 97, [arXiv: 1310.3545].

[14] A. A. Abdo, M. Ackermann, M. Ajello, et al., Fermi-LAT Observations of the Geminga Pulsar, ApJ 720 (Sept., 2010) 272-283. (Geminga). 